\title{
A Traffic Restriction Scheme for Enhancing Carpooling
}

\author{
Dong Ding and Bin Shuai \\ School of Transportation and Logistic, Southwest Jiaotong University, Chengdu 610031, China \\ Correspondence should be addressed to Bin Shuai; shuaibin@home.swjtu.edu.cn
}

Received 14 October 2016; Revised 23 January 2017; Accepted 20 February 2017; Published 12 March 2017

Academic Editor: Gabriella Bretti

Copyright ( 2017 Dong Ding and Bin Shuai. This is an open access article distributed under the Creative Commons Attribution License, which permits unrestricted use, distribution, and reproduction in any medium, provided the original work is properly cited.

For the purpose of alleviating traffic congestion, this paper proposes a scheme to encourage travelers to carpool by traffic restriction. By a variational inequity we describe travelers' mode (solo driving and carpooling) and route choice under user equilibrium principle in the context of fixed demand and detect the performance of a simple network with various restriction links, restriction proportions, and carpooling costs. Then the optimal traffic restriction scheme aiming at minimal total travel cost is designed through a bilevel program and applied to a Sioux Fall network example with genetic algorithm. According to various requirements, optimal restriction regions and proportions for restricted automobiles are captured. From the results it is found that traffic restriction scheme is possible to enhance carpooling and alleviate congestion. However, higher carpooling demand is not always helpful to the whole network. The topology of network, OD demand, and carpooling cost are included in the factors influencing the performance of the traffic system.

\section{Introduction}

Traffic congestion is becoming a world-wide problem. Several measures have emerged for alleviating traffic congestion, such as congestion pricing, signal control, and traffic restriction. Congestion pricing has been implemented in handful places such as Singapore, London, and Stockholm and received good performance as well as signal control and traffic restriction. However, they still come with respective undesirable affects. For example, congestion pricing often results in rejections from the public, signal control is difficult to implement in a large network, and normally traffic restriction inhibits travel demand to large extent so that it brings inconvenience to the population. Carpooling is a better choice owing to the relationship between vehicles and travelers. Namely, it is able to decrease the number of vehicles without reduced travel demands. Thus carpooling is supposed to be advocated for alleviating congestion.

However, a number of solo driving travelers remain preference to keep driving alone [1, 2]. Possible factors affecting travelers solo driving behavior have been revealed. As an example, Abrahamse and Keall [2] attributed the factors to some sociodemographic characteristics such as income, age, and gender. Teal [3] indicated that the travelers with longer travel distance and less access to a vehicle prefer carpooling. Vanoutrive et al. [4] analyzed the popularity of carpooling in Belgium and found that workplace location, organization, and promotion can explain carpooling behavior there. Manners such as providing carpooling information, HOV/HOT lanes, have been taken to prompt carpooling. Abrahamse and Keall [2] investigated the effect of webbased carpooling in New Zealand and manifested that the number of carpoolers had increased to $27 \%$ with carpooling information provided. Nevertheless, even in the presence of carpooling and measures prompting carpooling, the performance of traffic system seems not desirable in some cases. Kwon and Varaiya [5] argued that HOV lanes failed to attract more travelers to carpool so that overall congestion still exists. What is more, Dahlgren [6] showed that sometimes HOV lanes for carpooling perform worse than general purpose lanes. From the literatures above we attribute the main reason to insufficient share of carpooling travelers. As an alternative HOT lane policy shows a better performance than HOV lanes to some extent, especially in North America. Taking the I394 HOT lane as an example, Cho et al. [7] revealed that 
there are additional factors to influence time saving and the willingness of travelers to pay to use HOT lanes. Dahlgren [8] established a model to demonstrate the best situation of setting HOT lanes which is related to the initial delay and construct and operate cost. Thus HOT lane is not always a better choice to encourage carpooling to mitigate congestion. More effective measures need to be developed for enhancing carpooling, and the analysis of impacts of traffic restriction on traffic system is necessary.

Traffic restriction, as a scheme forbidding automobiles to enter the given regions, has been focused on in practice and theory. Daganzo [9] referred it as road spacing rationing and examined the effect of hybrid scheme of road spacing rationing and road pricing on the background of a bottleneck. Considering traffic equilibrium, the efficiency traffic restriction was analyzed $[9,10]$. Shi et al. [11] established formulations to capture the optimal district and restriction ratio in a real network. Traffic restriction has/had been implemented in many areas around the world, including Mexico City, Manila, Philippine, and Columbia. During 2008 Olympic Games, the traffic restriction scheme in Beijing received excellent performance in alleviating urban congestion and reducing traffic emission. Regardless of the visible effect, the drawbacks of traffic restriction are advocated. For example, Eskeland and Feyzioglu [12] pointed out that traffic restriction scheme failed in Mexico City since people always tried quite a few methods to avoid the restriction, such as purchasing additional vehicles, driving with other license plates or on weekends. Nie [13] indicated that traffic restriction scheme is a bad policy for social welfare, unless some complementary measures are taken, such as tradable credits scheme or control of vehicle quota as the travelers probably buy another vehicle with new road spacing qualification to avoid the regulation. However, purchasing another vehicle normally happens in the places with low price of owing a vehicle. It is a good policy under the condition that people cannot afford additional vehicles easily. Thus the impacts of traffic restriction scheme on efficiency need to be analyzed.

In conclusion, traffic restriction scheme needs complementary measures to optimize traffic system while carpooling needs schemes to prompt. Triggered by this, we propose a scheme imposing traffic restriction scheme to enhance carpooling. Namely, all restricted travelers have to either carpool to enter the restriction region or bypass it. Apparently the analysis of the performance of this scheme is necessary. For the purpose of minimizing total travel cost, the optimal scheme to capture the restriction region and proportion is designed.

The rest of this paper is organized as follows: Section 2 introduces the notations and assumptions of this paper and establishes the formulations of lower level. Section 3 investigates the impacts of various schemes to a simple network. The optimal scheme is designed in Section 4 with the demonstration of algorithm in Section 5 and applied to a Sioux Falls network example in Section 6. The conclusions and discussions are included in Section 7.

\section{Model Formulation}

2.1. Notation and Assumptions. Consider a strong connected network $(G, A)$. $G$ is the set of nodes, and $A$ is the set of links. $W$ and $M$ denote the set of OD pairs and travel modes, respectively. $L$ is the set of links.

We utilize the notation which represents the variables in this paper in the notation section.

For generality and simplicity, we adopt the three assumptions in this paper:

(1) There exits two travel modes in the network: solo driving and carpooling. We use $s$ and $c$ to represent the solo driving mode and carpooling mode, respectively. Thus $m=s, c$.

(2) According to the investigation [14], the average number of travelers in one vehicle is 1.6. Thus, in this paper, we assume two travelers in one vehicle for carpooling mode [15].

(3) Travelers follow the traffic restriction regulation. Namely, all the restricted travelers have to bypass the restriction region or carpool. Penalty cost does not exist in this paper because no one violates the restriction regulation.

2.2. Cost Function. Here we adopt the BPR function to compute the travel time.

$$
t_{a}=t_{a}^{0}\left[1+\beta\left(\frac{v_{a}^{c}}{C_{a}}\right)^{\alpha}\right], \quad a \in A .
$$

$\alpha$ and $\beta$ are constant parameters. The path cost of solo driving travelers can be measured by the path travel time. For carpooling travelers, carpooling cost on each path is unavoidable apart from the path travel time. We use $\Delta$ to represent the carpooling cost, which may include the waiting time, inconvenience, and the out-of-pocket savings and is set to be a constant [15]. Actually carpooling travelers will take the same time with solo driving travelers on a link. Thus the link cost function on link $a$ can be expressed as follows:

$$
c_{a}^{s}=c_{a}^{c}=\lambda_{t} \cdot t_{a}, \quad a \in A
$$

Carpooling cost will happen before a trip if travelers choose carpooling. Then the cost function on path $l$ can be denoted as follows:

$$
\begin{aligned}
& c_{w, l}^{s}=\lambda_{t} \cdot \sum_{a} t_{a} \cdot \delta_{a}^{l}, \quad a \in A, w \in W, l \in L, \\
& c_{w, l}^{c}=\Delta+\lambda_{t} \cdot \sum_{a} t_{a} \cdot \delta_{a}^{l}, \quad a \in A, w \in W, l \in L,
\end{aligned}
$$

where $\lambda_{t}$ is the value of time. For simplicity, in this paper we assume $\lambda_{t}$ is a constant, which means all travelers have the same value of time.

2.3. The Model of Mode Split and User Equilibrium. Based on the aforementioned assumptions, travelers choose either 
solo driving or carpooling. The share of modes can be split by Logit formulation:

$$
d_{w}^{m}=d_{w} \cdot \frac{\exp \left(-\theta \mu_{w}^{m}\right)}{\sum_{m} \exp \left(-\theta \mu_{w}^{m}\right)}, \quad w \in W, m \in M
$$

where the $\mu_{w}^{m}$ is the minimal cost of travel mode $m$ between OD pair $w$, and $\theta$ is the sensitive coefficient.

In a network with traffic restriction solo driving travelers have to bypass the district or carpool since they are forbidden to enter the restriction region. When there is no path connecting the OD pair out of the region, they only have carpooling choice. Similar to [11, 13], the minimal cost function of solo driving travelers between the OD pair $w$ in which the restricted travelers are not fully blocked can be calculated as follows:

$$
\mu_{w}^{s}=(1-\gamma) \mu_{w}^{s u}+\gamma \mu_{w}^{s r}, \quad w \in W,
$$

where $\mu_{w}^{s u}$ represents the minimal cost of unrestricted solo driving travelers and $\mu_{w}^{s r}$ denotes the minimal cost of restricted solo driving travelers. $\gamma$ is the restriction proportion of automobiles.

Some OD pairs, which are denoted as $W^{r}$, are not connected by any path which is out of the restriction region. Namely, the travelers between these OD pairs have to carpool since solo driving travelers cannot pass the region. The minimal cost function of the travelers between $W^{r}$ is as follows:

$$
\mu_{w}^{s}=(1-\gamma) \mu_{w}^{s u}+\gamma \mu_{w}^{c}, \quad w \in W^{r} .
$$

The minimal cost function of carpooling travelers is

$$
\mu_{w}^{c}=\min \left(c_{w, l}^{c}\right), \quad w \in W, l \in L
$$

Then the following variational inequality (VI) program is organized to express the mode split and user equilibrium:

$$
\begin{aligned}
& \sum_{w \in W} \sum_{l \in L} c_{w, l}^{c}\left(f^{*}\right)\left(f_{w, l}^{c}-f_{w, l}^{c *}\right) \\
& +\sum_{w \in W} \sum_{l \in L} c_{w, l}^{s u}\left(f^{*}\right)\left(f_{w, l}^{s u}-f_{w, l}^{s u *}\right) \\
& +\sum_{w \in W^{r}} \sum_{l \in L} c_{w, l}^{s r}\left(f^{*}\right)\left(f_{w, l}^{s r}-f_{w, l}^{s r *}\right) \\
& +\sum_{w \in W} \frac{1}{\theta} \ln \frac{d_{w}^{c *}}{d_{w}}\left(d_{w}^{c}-d_{w}^{c *}\right) \\
& +\sum_{w \in W} \frac{1}{\theta} \ln \frac{d_{w}^{s *}}{d_{w}}\left(d_{w}^{s}-d_{w}^{s *}\right) \geq 0 .
\end{aligned}
$$

The solutions $\left(f_{w, l}^{c *}, f_{w, l}^{s u *}, f_{w, l}^{s r *}, d_{w}^{c *}, d_{w}^{s *}\right)$ belong to the following feasible region:

$$
\begin{aligned}
& d_{w}^{c}+d_{w}^{s}=d_{w}, \quad w \in W, \\
& \sum_{l \in L} f_{w, l}^{s u}=(1-\gamma) d_{w}^{s}, \quad w \in W, \\
& \sum_{l \in L} f_{w, l}^{s r}=\gamma d_{w}^{s}, \quad w \in W^{r}, \\
& \sum_{l \in L} f_{w, l}^{c}=d_{w}^{c}, \quad w \in W, \\
& f_{w, l}^{s r} \geq 0, \quad w \in W^{r}, \quad l \in L, \\
& f_{w, l}^{s u} \geq 0, \quad w \in W, \quad l \in L, \\
& f_{w, l}^{c} \geq 0, \quad w \in W, \quad l \in L .
\end{aligned}
$$

The complementarity conditions are

$$
\begin{aligned}
f_{w, l}^{i} \cdot \lambda_{w}^{i}=0, \quad i=(c, s u), w \in W, l \in L, \\
\lambda_{w}^{i} \geq 0, \quad i=(c, s u), w \in W, \\
f_{w, l}^{s r} \cdot \lambda_{w}^{s r}=0, \quad w \in W^{r}, \quad l \in L, \\
\lambda_{w}^{s r} \geq 0, \quad w \in W^{r} .
\end{aligned}
$$

Proposition 1. The VI program above is equivalent to Logit mode split and user equilibrium.

Proof. According to KKT conditions, we have the following equations:

$$
\begin{gathered}
f_{w, l}^{c}: c_{w, l}^{c}-\mu_{w}^{c}-\lambda_{w}^{c}=0, \quad w \in W, \quad l \in L, \\
f_{w, l}^{s u}: c_{w, l}^{s u}-\mu_{w}^{s u}-\lambda_{w}^{s u}=0, \quad w \in W, \quad l \in L, \\
f_{w, l}^{s r}: c_{w, l}^{s r}-\mu_{w}^{s r}-\lambda_{w}^{s r}=0, \quad w \in W^{r}, \quad l \in L, \\
d_{w}^{c}: \frac{1}{\theta} \ln \frac{d_{w}^{c}}{d_{w}}-\omega_{w}+\mu_{w}^{c}, \quad w \in W, \\
d_{w}^{s}: \frac{1}{\theta} \ln \frac{d_{w}^{s}}{d_{w}}-\omega_{w}+(1-\gamma) \mu_{w}^{s u}+\gamma \mu_{w}^{s r}, \quad w \in W .
\end{gathered}
$$

From equations (16) and (22), we obtain the following equations:

$$
\begin{aligned}
& c_{w, l}^{c}=\mu_{w}^{c}, \quad \text { if } f_{w, l}^{c}>0, w \in W, l \in L, \\
& c_{w, l}^{c} \geq \mu_{w}^{c}, \quad \text { if } f_{w, l}^{c}=0, w \in W, l \in L, \\
& c_{w, l}^{s u}=\mu_{w}^{s u}, \quad \text { if } f_{w, l}^{s u}>0, w \in W, l \in L, \\
& c_{w, l}^{s u} \geq \mu_{w}^{s u}, \quad \text { if } f_{w, l}^{s u}=0, w \in W, l \in L, \\
& c_{w, l}^{s r}=\mu_{w}^{s r}, \quad \text { if } f_{w, l}^{s r}>0, w \in W^{r}, l \in L, \\
& c_{w, l}^{s r} \geq \mu_{w}^{s r}, \quad \text { if } f_{w, l}^{s r}=0, w \in W^{r}, l \in L .
\end{aligned}
$$


The equations above imply that the route choice of users follows user equilibrium, which means travelers always choose the route with the minimal travel cost, and no individual can curtain his/her travel cost by changing the route.

From equations (23) and (24), we can obtain the Logit formulation. Hence, the VI program meets the Logit mode split and user equilibrium.

When the restriction region is given, the feasible path sets of solo driving travelers and carpooling travelers are fixed. The feasible region of the VI program is nonnegative and linear while the VI formulation is continuous. It means that there exists at least one solution for the VI program (8). Similar specific proof can be seen in $[11,24]$.

Several approaches have emerged for solving VI program, such as Frank-Wolfe algorithm, projection algorithm, and sensitive-based analysis algorithm. Here, the block GaussSeidel decomposition approach together with the method of successive averages is applied to aforementioned VI program. In terms of this approach the demands of travel modes and flow patterns can be obtained.

\section{Impacts of Traffic Restriction Scheme}

In this paper we adopt the total travel cost, which is expressed later, to measure the performance of a network.

$$
Z=\Delta \cdot \sum_{w \in W} d_{w}^{c}+\sum_{a \in A}\left(c_{a}^{s} \cdot v_{a}^{s}+c_{a}^{c} \cdot v_{a}^{c}\right)
$$

For a traffic restriction scheme two components have to be decided: the traffic restriction region and proportion. With various regions and proportions, the performances of networks are different. In addition, other factors such as carpooling cost can influence the efficiency of the scheme. We explore the impacts of a simple network under the scheme. The simple network with six nodes and seven links is illustrated in Figure 1. The OD demands are listed later as well as the attributes of links in Tables 1 and 2. $\theta=0.05, \lambda_{t}=$ 2, $\alpha=4$, and $\beta=0.15$.

Here are four types of scenarios: no traffic restriction, the whole network region with proportion 1, given part of the network region with the proportion 1 , and given part of the network region with given proportion less than 1 . Table 3 shows the results under the four scenarios. With the same restriction region, the total travel cost under scheme 3 is lower than that under scheme 4 owing to more carpooling travelers. Scheme 1 without traffic restriction and scheme 2 with all restriction links and restricted solo driving travelers have the highest and lowest total travel cost, respectively.

Figure 2 displays the respective trend of total travel cost under the four scenarios with various carpooling costs. It is apparent that when carpooling cost increases, all the total travel costs increase. Note that at the beginning the total travel cost under Scenario 2 is the lowest and the highest one is under Scenario 1. However, the curve under Scenario 2 has the fastest growing rate and the slowest growing rate follows Scenario 1 . When the carpooling cost equals 1.5 , the total travel cost under Scenario 2 begins to be less than that under

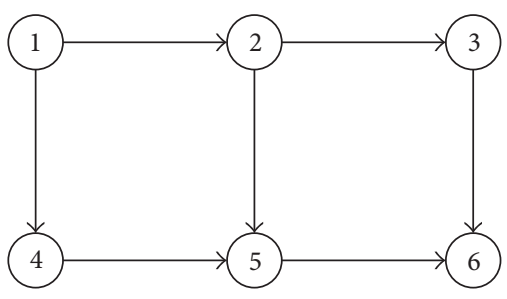

FIGURE 1: A simple network.

Scenario 3. The explanation is that when all the travelers are forbidden to enter the restriction region, they have to carpool, leading to higher total carpooling cost. So the total travel cost still increases, although the travel time on links decreases. When the carpooling cost equals 2.5, the total travel cost under Scenario 4 is the maximal, which means the carpooling cost plays the main role in the total travel cost. When the carpooling cost equals 5, the total travel cost without traffic restriction scheme is minimal. Thus if the carpooling cost is too high, the best choice is to abandon traffic restriction.

Figure 3 shows the total travel costs with various carpooling costs and proportion when links 1 and 2 are restricted. The total travel cost increases generally with the increasing carpooling cost. At some points, the total travel costs with smaller proportions are less than those with larger proportion. For example, when the carpooling cost varies from 3 to 5 , the total travel cost with proportion 0.3 is lower than that with proportion 0.4 . From Figures 2 and 3 we know that the total travel cost is associated with factors such as topology of the network, travel demands, carpooling cost, and traffic restriction scheme.

\section{Optimal Traffic Restriction Design}

Under user equilibrium, no travelers can curtail their travel costs by changing routes, which can be described as a Nash equilibrium. By implementing a traffic restriction scheme, our target is to minimize the total travel cost under the equilibrium state. A bilevel program is applied and the upper level is organized as follows:

$$
\begin{array}{ll}
\min & Z=\Delta \cdot \sum_{w \in W} d_{w}^{c}+\sum_{a \in A}\left(c_{a}^{s} \cdot v_{a}^{s}+c_{a}^{c} \cdot v_{a}^{c}\right), \\
\text { s.t. } & 0 \leq \gamma \leq 1 .
\end{array}
$$

The lower level is the variational inequality (10), and the flow patterns can be obtained from it.

In terms of the objective, the selection of traffic restriction links is a conventional problem. However, the restriction links do not always assemble a connected region. In real circumstances, road managers normally select an area as a traffic restriction region for convenience of implementation and the effect of alleviating congestion. And if a special event will happen in a place, the region including this place is expected to be uncongested. Thus this place is supposed to be included in the restriction region. In addition, in many cities the restriction proportion is plate-number-based. The common methods are odd-and-even license plate rule and 
TABLE 1: OD demands.

\begin{tabular}{lccccccc}
\hline OD pair & $1-2$ & $1-6$ & $2-3$ & $2-5$ & $3-6$ & $4-5$ & $5-6$ \\
\hline Demand (veh/h) & 1000 & 2000 & 1000 & 800 & 600 & 1200 & 500 \\
\hline
\end{tabular}

TABLE 2: Attributes of links.

\begin{tabular}{lccccccc}
\hline Link & $1-2$ & $2-3$ & $1-3$ & $2-5$ & $3-6$ & $4-5$ & $5-6$ \\
\hline$t_{a}^{0}(\min )$ & 6 & 4 & 3 & 3 & 3 & 4 & 2 \\
$C_{a}(\mathrm{veh} / \mathrm{h})$ & 2000 & 2000 & 1000 & 1000 & 1000 & 2000 & 2000 \\
\hline
\end{tabular}

TABle 3: Performance of scenarios.

\begin{tabular}{lcccc}
\hline & Scenario 1 & Scenario 2 & Scenario 3 & Scenario 4 \\
\hline Restriction links & none & all & 1,2 & 1,2 \\
Proportion & 0 & 1 & 1 & 0.3 \\
Total travel cost & 90276 & 83186 & 84192 & 89299 \\
Carpooling demand & 3506 & 7100 & 5252 & 4033 \\
\hline
\end{tabular}

tail number restriction. Thus the proportion can be 0.5 (oddand-even license plate rule), 0.2 (every two tail numbers per day among workdays), and 0.1 (every tail number in turn). For these reasons, we detect the scenarios that the links always construct a connected region and with fixed proportions. Then we will use an example to demonstrate the performance.

\section{Algorithm}

Nonconvex property makes it difficult to solve the problem although some relevant algorithms are proposed. Our target is to capture the optimal district and the proportion. For the optimization of district, almost all the previous studies denote $n_{a}$ the binary variable to decide whether a link is selected. If link a is selected, $n_{a}$ equals 1 , otherwise 0 , which is very similar to the discrete network design problem (DNDP). Besides, continuous network design (CNDP) enlightens us with the method to obtain the proportion. Thus we are able to take mixed network design problem (MNDP) as the reference. Table 4 lists some algorithms of network design problem with the expression of their superiorities and drawbacks. It displays that some algorithms are convenient to implement without guarantee of global optimal solutions while some of them are helpful for the global optimal solutions but computationally demanding. For a large-scale network, it might be extremely difficult to utilize the algorithms which capture global optimal solutions due to the very expensive computation, such as Branch and Bound algorithm [20] and simulation [25]. In addition, the difference between our problem and MNDP is that the optimal area is required to be connected, which may induce impossibility of some of the algorithms. Friesz et al. [25] pointed out that only when the global optimal solution or near global optimal solution is essential can we adopt their algorithm. In this paper, the objective is minimization of total travel cost and even a local optimal solution can provide reference to the managers. Zhang and Yang [26] proposed a genetic-based algorithm for optimal congestion pricing cordon and charge

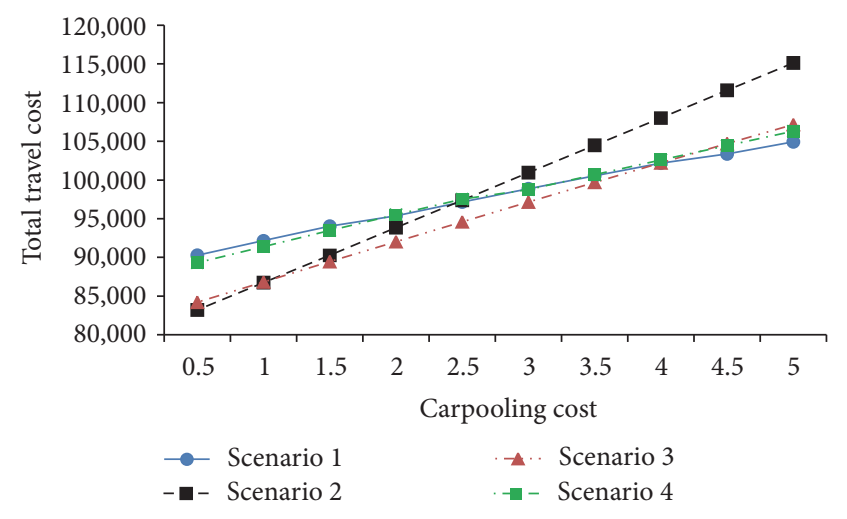

FIGURE 2: The total travel costs with various carpooling costs.

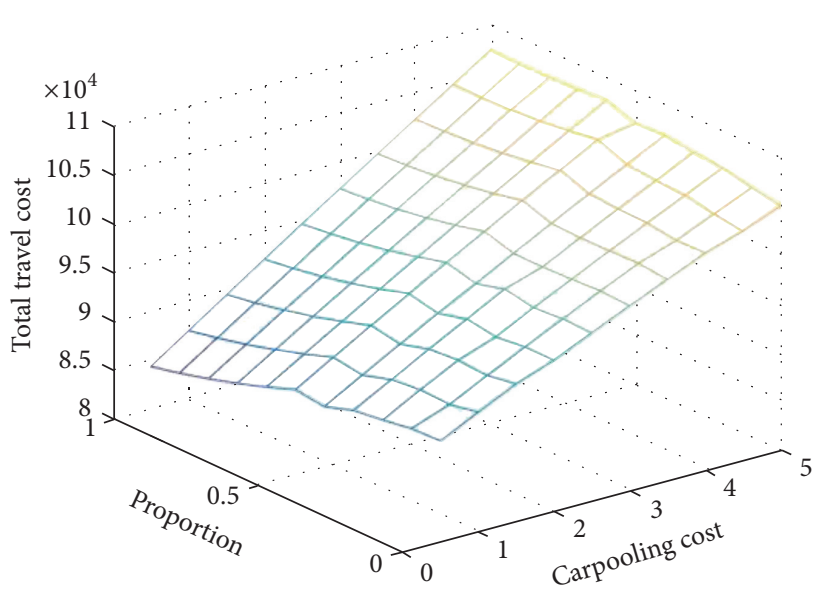

FIGURE 3: The total travel costs with various carpooling costs and proportions.

simultaneously in a large-size realistic network. Thus for the feasibility and convenience, we adopt the genetic algorithm to optimize the traffic restriction area and the proportion simultaneously (see also [11]).

\section{Numerical Example}

Here we take the Sioux Falls network presented in [27] as an example to design the optimal traffic restriction scheme in the presence of carpooling. It is assumed that the influence of the pair of links between any two nodes is not mutual. The 
TABLE 4: Algorithms of network design problem.

\begin{tabular}{|c|c|c|c|c|}
\hline Approach & Proposer/user & Case & Superiorities & Drawbacks \\
\hline Ant system & $\begin{array}{l}\text { Poorzahedy and } \\
\text { Abulghasemi [16] }\end{array}$ & Discrete network design & $\begin{array}{l}\text { Less computational demand and } \\
\text { simple implementation }\end{array}$ & $\begin{array}{c}\text { No guarantee of global optimal } \\
\text { solution }\end{array}$ \\
\hline Genetic algorithm & $\begin{array}{c}\text { Drezner and } \\
\text { Wesolowsky [17] }\end{array}$ & Discrete network design & $\begin{array}{l}\text { Less computational demand and } \\
\text { simple implementation }\end{array}$ & $\begin{array}{l}\text { No guarantee of global optimal } \\
\text { solution }\end{array}$ \\
\hline $\begin{array}{l}\text { Hybrid } \\
\text { meta-heuristic } \\
\text { algorithm }\end{array}$ & $\begin{array}{l}\text { Poorzahed and } \\
\text { Rouhani [18] }\end{array}$ & Discrete network design & $\begin{array}{l}\text { Less computational demand and } \\
\text { simple implementation }\end{array}$ & $\begin{array}{c}\text { No guarantee of global optimal } \\
\text { solution }\end{array}$ \\
\hline Support fuction & Gao et al. [19] & Discrete network design & Global optimal solution & Expensive computation \\
\hline $\begin{array}{l}\text { Branch and Bound } \\
\text { algorithm }\end{array}$ & $\begin{array}{l}\text { Farvaresh and } \\
\text { Sepehri }[20]\end{array}$ & Discrete network design & Global optimal solution & Expensive computation \\
\hline Simulation & Friesz et al. (1992) & $\begin{array}{l}\text { Continuous network } \\
\text { design }\end{array}$ & $\begin{array}{l}\text { Global optimal solution or being } \\
\text { very close to global optimal } \\
\text { solution }\end{array}$ & Expensive computation \\
\hline $\begin{array}{l}\text { SO-relaxation } \\
\text { based method and } \\
\text { UE-reduction } \\
\text { based method }\end{array}$ & Wang et al. [21] & Discrete network design & Global optimal solution & Expensive computation \\
\hline $\begin{array}{l}\text { Global optimal } \\
\text { approach }\end{array}$ & Wang and Lo [22] & Mixed network design & Global optimal solution & $\begin{array}{l}\text { More restraints and expensive } \\
\text { computation }\end{array}$ \\
\hline $\begin{array}{l}\text { MILP formulation } \\
\text { approach }\end{array}$ & Luathep et al. [23] & Mixed network design & Global optimal solution & Expensive computation \\
\hline
\end{tabular}

attributes of links and the demands are the same with those in [27].

Several scenarios exist when implementing traffic restriction. In this example, we propose three scenarios.

Scenario 1. Normally the traffic restriction links constitute a connected region. In this scenario the links in an optimal connected region and the proportion are captured.

Scenario 2. Sometimes the uncongested places are necessary due to some reasons, for example, when important event happens. In this scenario we assume that node 10 is a required uncongested place. Namely, all the traffic restriction schemes are supposed to include node 10 . Then we explore the optimal region and proportion as well as the performance of the network.

Scenario 3. For the sake of feasibility, not all methods can be implemented in realistic world. Thus in this scenario we assume the restriction proportion is 0.5 and 0.2 , respectively. Then we capture the optimal region and detect the performance.

Scenario 4 is the situation without traffic restriction. Figure 4 illustrates the traffic restriction regions of scenarios. All regions are connected and a part of the whole network, which means the scenarios are convenient to be implemented in realistic world. Table 5 lists the performances of the four scenarios. Under various scenarios, total travel costs under various scenarios are less than that without traffic restriction as well as the numbers of vehicles, and the carpooling demands are higher than that. It displays that the traffic restriction is able to prompt carpooling and thereby alleviate congestion. Scenario 1 has the least total travel cost, which shows that our model and algorithm are effective. Scenario 2 includes node 10 , which matches the requirement that node 10 is supposed to be in the restriction region. In terms of the predefined restriction proportion, the optimal region is captured in the case of Scenario 3. It demonstrates that the scenarios with the requirements of specific region or proportion to encourage travelers to carpool by traffic restriction are feasible. It is worth noticing that in Table 5 the carpooling demand under Scenario 1 is even less than that under Scenario 2 and the vehicles under Scenario 1 are more than that under Scenario 2, although the total travel cost under Scenario 1 is less than that under Scenario 2. This is because the areas give rise to different flow patterns leading to different carpooling demand, number of vehicles, and total travel costs. It demonstrates that higher carpooling demand is not always beneficial to the network. Although the restriction region under Scenario 1 is the same as that under Scenario 3(1), the different proportions lead to different total travel costs. Besides, the proportion under Scenario 1 is less than that under Scenario 3(1). It proves again that the performance depends on various factors such as carpooling cost, topology of network, and demand.

Table 6 lists the weight effects on the components of total travel costs under various scenarios. It is shown that the weights are similar. Since the carpooling cost is low, the costs of total travel time play the main roles while the costs of carpooling are just small parts of the total travel costs. However, carpooling cost still impact the mode choice due to the scale of the network and the demand. Namely, a large scale of network with a certain quantity of carpoolers may generate the corresponding total travel time and thereby influences the performance of the whole network, although the total car- 


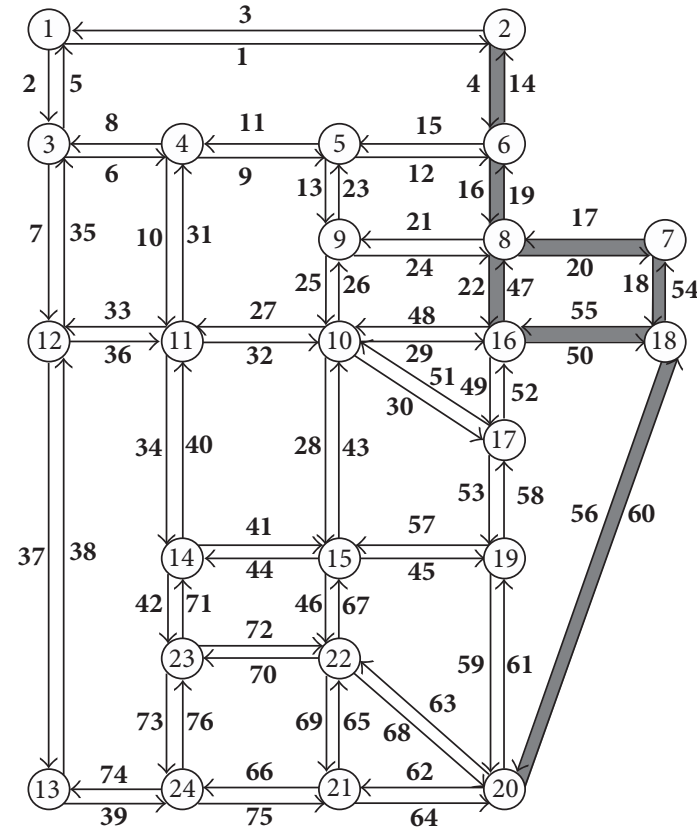

(a) The region under Scenario 1

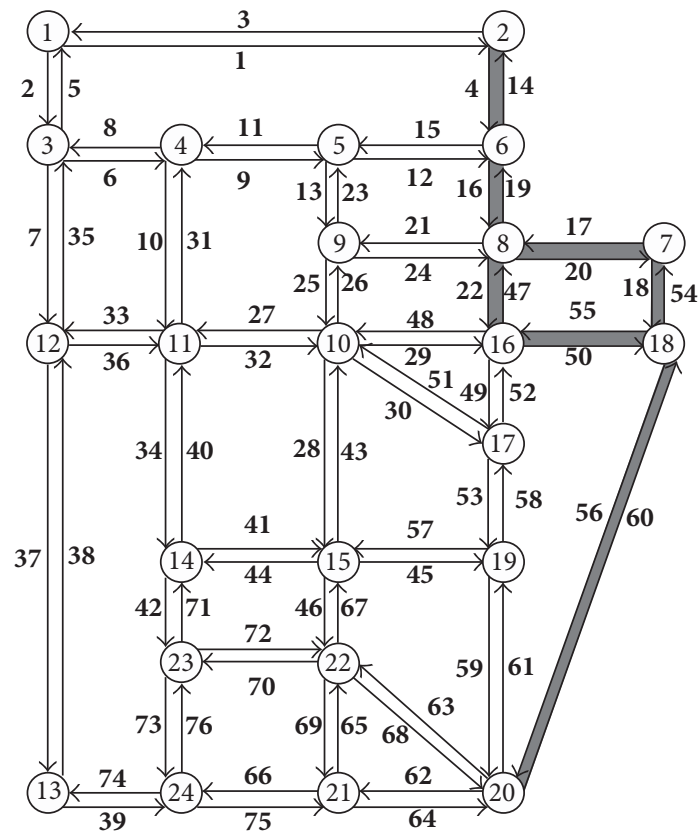

(c) The region under Scenario 3 with proportion 0.5

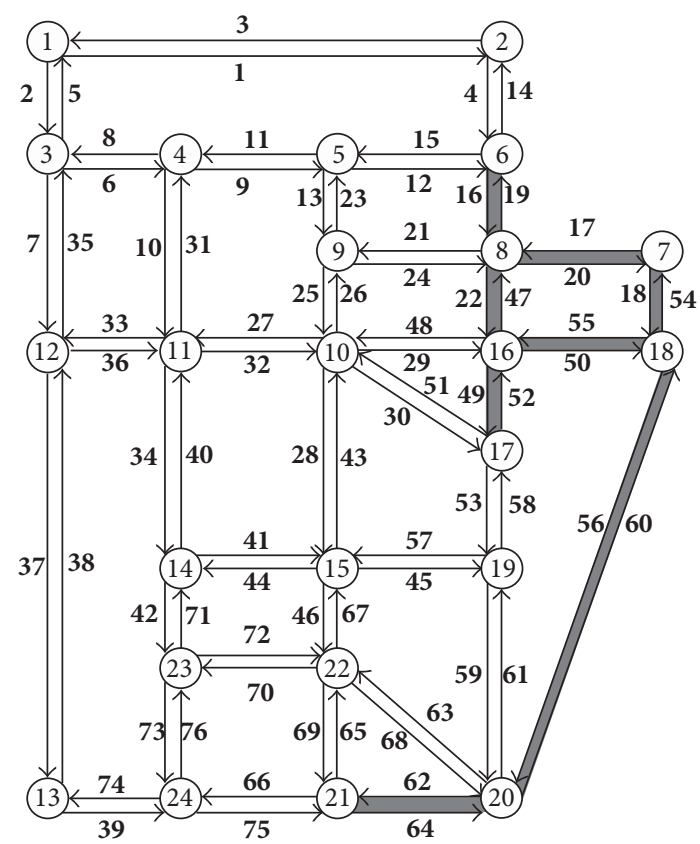

(b) The region under Scenario 2

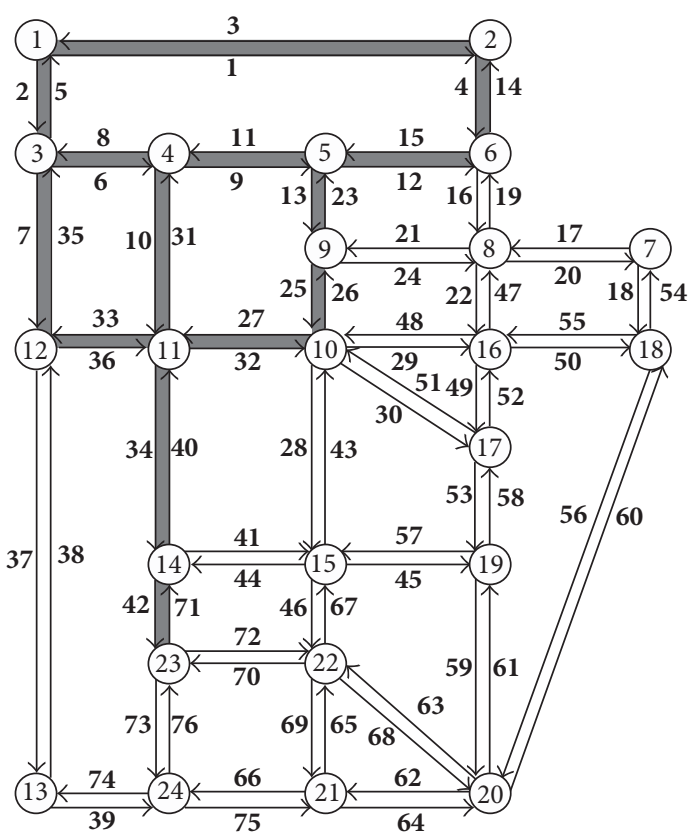

(d) The region under Scenario 3 with proportion 0.2

FIGURE 4: The traffic restriction regions under various scenarios.

pooling cost is lower. Therefore attributes of network, demand, and carpooling cost cannot be ignored.

\section{Conclusion}

For the purpose of prompting carpooling, this paper proposes a scheme that using traffic restriction for solo driving travelers to encourage them to carpool. By a variational inequality we examine the impacts of this scheme on carpooling in a simple network with fixed restriction links and proportions; then taking Sioux falls network as an example we design the optimal scheme to capture the optimal region and proportion under various scenarios. The algorithm based on genetic algorithm is adopted for the solution. The results show that this scheme can encourage more travelers to carpool and mitigate congestion with appropriate restriction links/region 
TABLE 5: Performances of the scenarios.

\begin{tabular}{lccccc}
\hline & Scenario 1 & Scenario 2 & Scenario 3(1) & Scenario 3(2) & No scenario \\
\hline Proportion & 0.28 & 0.34 & 0.5 & 0.2 & - \\
Total travel cost & 365876 & 367071 & 366515 & 369409 & 370956 \\
Carpooling demand & 10162 & 10539 & 9715 & 9387 & 8681 \\
Number of vehicles & 12957 & 12769 & 13180 & 13344 & 13698 \\
\hline
\end{tabular}

TABLE 6: Weight effects on components of total travel costs under the scenarios.

\begin{tabular}{lccccc}
\hline & Scenario 1 & Scenario 2 & Scenario 3(1) & Scenario 3(2) & No scenario \\
\hline Weight of time cost (\%) & 95.83 & 95.69 & 96.02 & 96.19 & 96.49 \\
Weight of carpooling cost (\%) & 4.17 & 4.31 & 3.98 & 3.81 & 3.51 \\
\hline
\end{tabular}

and proportion, which shows the feasibility of this scheme and the positive effects in realistic cases. If the carpooling cost is relatively too high, normally this scheme cannot bring benefits. It is worth noticing that higher carpooling demand may result in more total travel time. In addition, not only carpooling cost but also topology of network and travel demand are the factors influencing the performance of the network. Hence we need to take into account the essential factors to analyze the specific cases.

In this paper we just consider the travel time and carpooling cost as the components of cost function and the minimal total travel cost objective. The future direction includes more factors for cost function and more objectives, for example, environment and economics.

\section{Notation}

$t_{a}^{0}: \quad$ The free flow travel time on link $a, a \in A$

$t_{a}$ : The travel time on link $a, a \in A$

$C_{a}$ : The capacity on link $a, a \in A$

$d_{w}$ : The travel demand between OD pair $w, w \in W$

$d_{w}^{m}$ : The travel demand of mode $m$ between OD pair $w, w \in W, m \in M$

$\triangle$ : Carpooling cost

$f_{w, l}^{m}$ : The traffic flow of mode $m$ on path $l$ between OD pair $w, w \in W, m \in M, l \in L$

$v_{a}^{m}$ : The traveler flow of mode $m$ on link $a, m \in M, a \in A$

$v_{a}^{c}$ : The vehicle flow on $\operatorname{link} a, a \in A$.

\section{Conflicts of Interest}

The authors declare that they have no conflicts of interest regarding the publication of this paper.

\section{References}

[1] M. V. Vugt, "Commuting by car or public transportation? A social dilemma analysis of travel mode judgements," Europe Journal of Operation Research, vol. 26, pp. 373-395, 1996.

[2] W. Abrahamse and M. Keall, "Effectiveness of a web-based intervention to encourage carpooling to work: A Case Study of Wellington, New Zealand," Transport Policy, vol. 21, pp. 45-51, 2012.
[3] R. F. Teal, "Carpooling: who, how and why," Transportation Research Part A: General, vol. 21, no. 3, pp. 203-214, 1987.

[4] T. Vanoutrive, E. Van De Vijver, L. Van Malderen et al., "What determines carpooling to workplaces in Belgium: location, organisation, or promotion?" Journal of Transport Geography, vol. 22, pp. 77-86, 2012.

[5] J. Kwon and P. Varaiya, "Effectiveness of California's High Occupancy Vehicle (HOV) system," Transportation Research Part C, vol. 16, no. 1, pp. 98-115, 2008.

[6] J. Dahlgren, "High occupancy vehicle lanes: not always more effective than general purpose lanes," Transportation Research Part A: Policy and Practice, vol. 32, no. 2, pp. 99-114, 1998.

[7] Y. Cho, R. Goel, P. Gupta, G. Bogonko, and M. W. Burris, "What are I-394 HOT lane drivers paying for?" in Proceedings of the 90th Annual Meeting Transportation Research Board (TRB '11), 2011.

[8] J. Dahlgren, "High-occupancy/toll lanes: where should they be implemented?" Transportation Research Part A: Policy and Practice, vol. 36, no. 3, pp. 239-255, 2002.

[9] C. F. Daganzo, "A pareto optimum congestion reduction scheme," Transportation Research Part B, vol. 29, no. 2, pp. 139154, 1995.

[10] D. Han, H. Yang, and X. Wang, "Efficiency of the plate-numberbased traffic rationing in general networks," Transportation Research Part E: Logistics and Transportation Review, vol. 46, no. 6, pp. 1095-1110, 2010.

[11] F. Shi, G.-M. Xu, B. Liu, and H. Huang, "Optimization method of alternate traffic restriction scheme based on elastic demand and mode choice behavior," Transportation Research Part C: Emerging Technologies, vol. 39, pp. 36-52, 2014.

[12] G. S. Eskeland and T. Feyzioglu, "Rationing can backfire: the "day without a car" in Mexico City," World Bank Economic Review, vol. 11, no. 3, pp. 383-408, 1997.

[13] M. Y. Nie, "Why license plate rationing is a bad transport policy?" Working Paper.

[14] Bundesamt für Statistik, Mobilitt in der Schweiz Ergebnisse des Mikrozensus Mobilitt und Verkehr 2010, 2012.

[15] H. Yang and H.-J. Huang, "Carpooling and congestion pricing in a multilane highway with high-occupancy-vehicle lanes," Transportation Research Part A: Policy and Practice, vol. 33, no. 2, pp. 139-155, 1999.

[16] H. Poorzahedy and F. Abulghasemi, "Application of Ant System to network design problem," Transportation, vol. 32, no. 3, pp. 251-273, 2005.

[17] Z. Drezner and G. O. Wesolowsky, "Network design: selection and design of links and facility location," Transportation 
Research Part A: Policy and Practice, vol. 37, no. 3, pp. 241-256, 2003.

[18] H. Poorzahedy and O. M. Rouhani, "Hybrid meta-heuristic algorithms for solving network design problem," European Journal of Operational Research, vol. 182, no. 2, pp. 578-596, 2007.

[19] Z. Gao, J. Wu, and H. Sun, "Solution algorithm for the bi-level discrete network design problem," Transportation Research Part B: Methodological, vol. 39, no. 6, pp. 479-495, 2005.

[20] H. Farvaresh and M. M. Sepehri, "A branch and bound algorithm for bi-level discrete network design problem," Networks and Spatial Economics, vol. 13, no. 1, pp. 67-106, 2013.

[21] S. Wang, Q. Meng, and H. Yang, "Global optimization methods for the discrete network design problem," Transportation Research Part B: Methodological, vol. 50, pp. 42-60, 2013.

[22] D. Z. W. Wang and H. K. Lo, "Global optimum of the linearized network design problem with equilibrium flows," Transportation Research Part B: Methodological, vol. 44, no. 4, pp. 482-492, 2010.

[23] P. Luathep, A. Sumalee, W. H. K. Lam, Z.-C. Li, and H. K. Lo, "Global optimization method for mixed transportation network design problem: a mixed-integer linear programming approach," Transportation Research Part B: Methodological, vol. 45, no. 5, pp. 808-827, 2011.

[24] J. Zhu, F. Xiao, and X. Liu, "Taxis in road pricing zone: should they pay the congestion charge?" Journal of Advanced Transportation, vol. 49, no. 1, pp. 96-113, 2015.

[25] T. L. Friesz, H.-J. Cho, N. J. Mehta, R. L. Tobin, and G. Anandalingam, "Simulated annealing approach to the network design problem with variational inequality constraints," Transportation Science, vol. 26, no. 1, pp. 18-26, 1992.

[26] X. Zhang and H. Yang, "The optimal cordon-based network congestion pricing problem," Transportation Research Part B: Methodological, vol. 38, no. 6, pp. 517-537, 2004.

[27] C.-Y. Yan, R. Jiang, Z.-Y. Gao, and H. Shao, "Effect of speed limits in degradable transport networks," Transportation Research Part C: Emerging Technologies, vol. 56, pp. 94-119, 2015. 


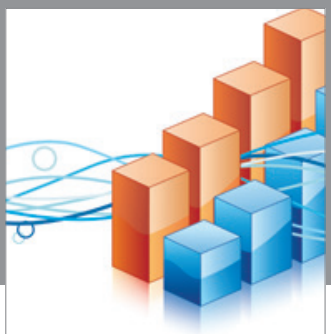

Advances in

Operations Research

vatem alat4

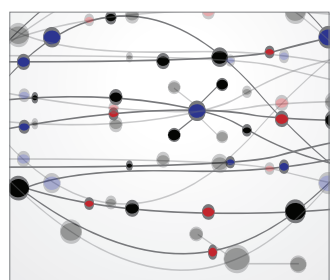

\section{The Scientific} World Journal
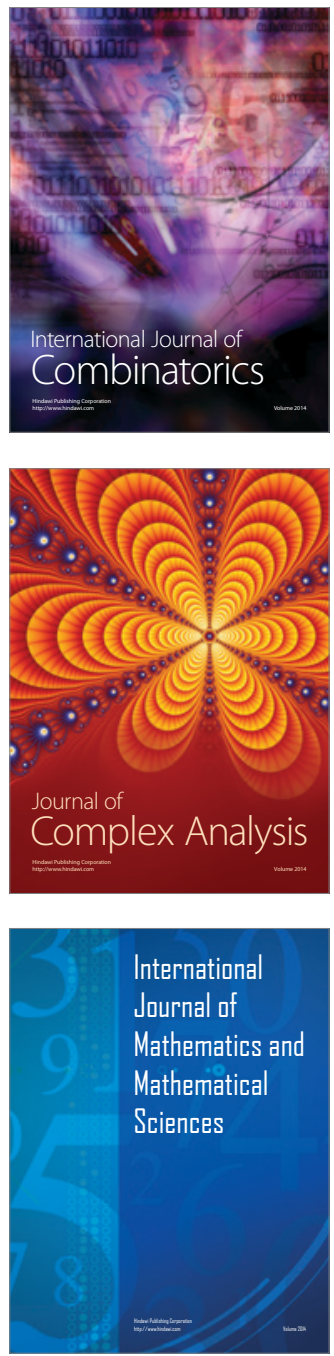
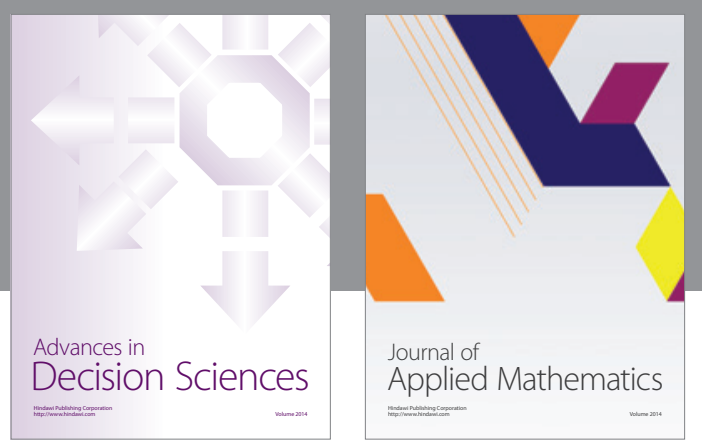

Algebra

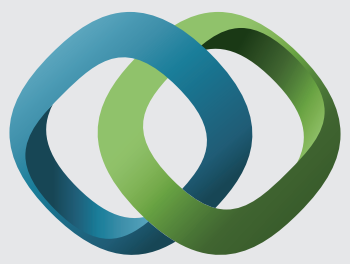

\section{Hindawi}

Submit your manuscripts at

https://www.hindawi.com
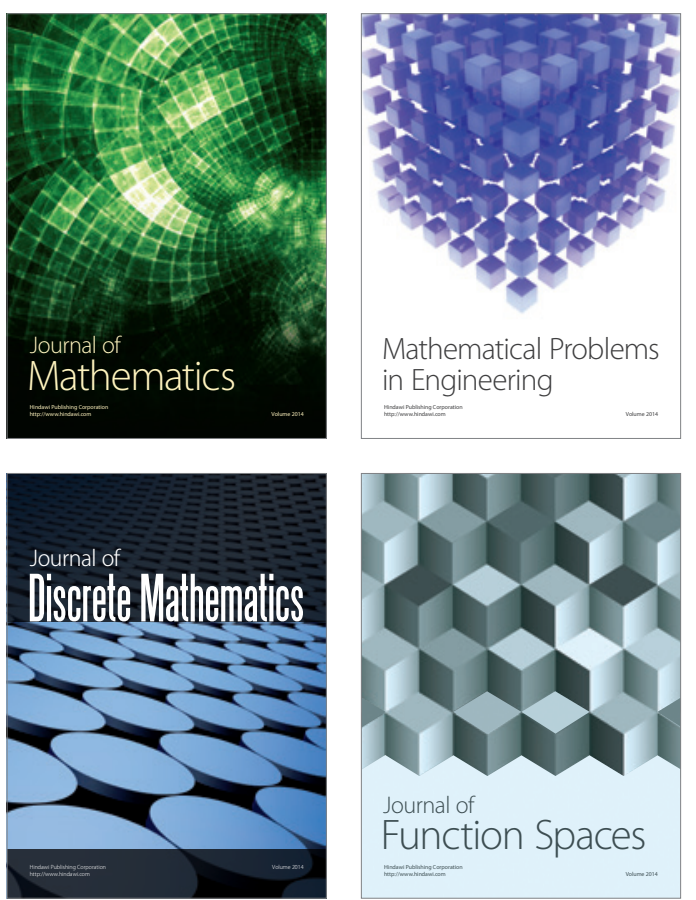

Mathematical Problems in Engineering
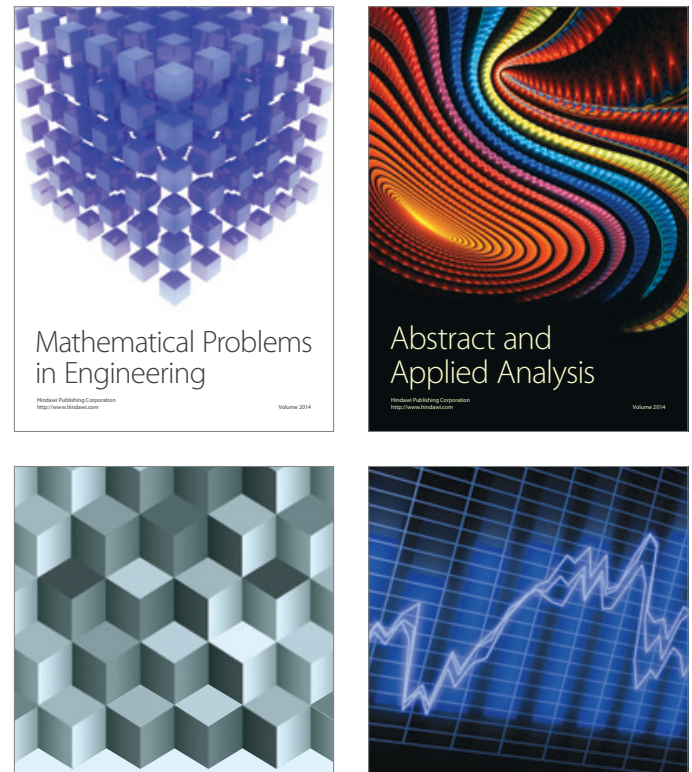

Journal of

Function Spaces

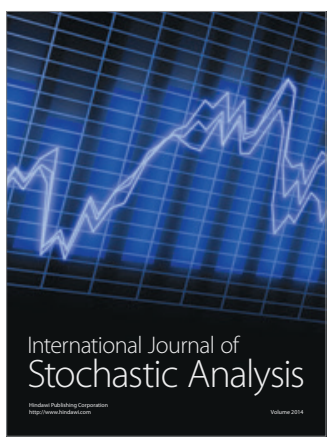

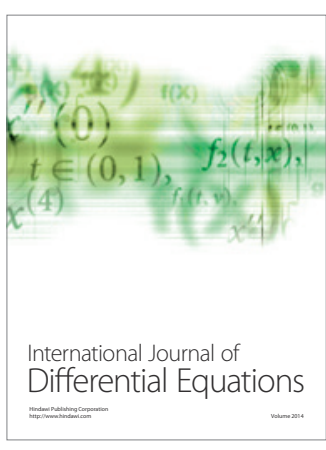
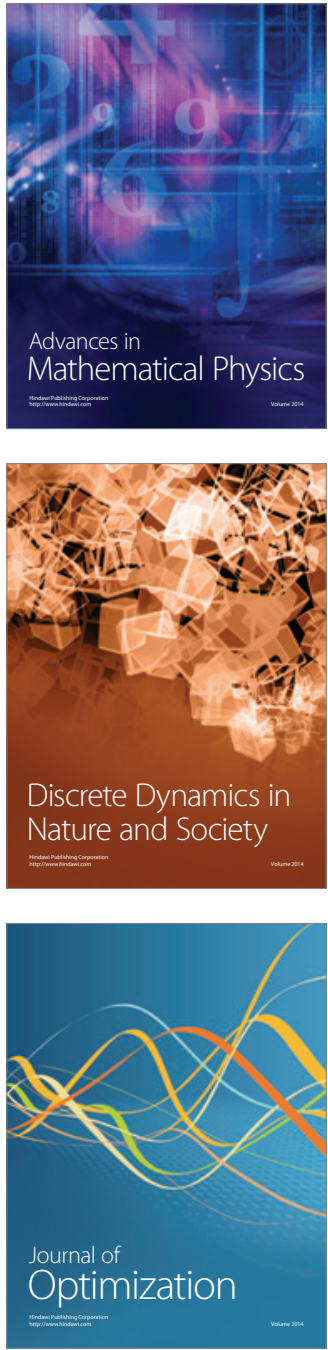